\title{
Septische Granulomatose als seltene Differenzialdiagnose einer chronisch-entzündlichen Darmerkrankung
}

\section{Chronic granulomatous disease as a rare differential diagnosis of inflammatory bowel disease}

\author{
Autoren \\ Tim Zimmermann ${ }^{1}$ \\ Institute \\ 1 I. Medizinische Klinik und Poliklinik, Gastroenterologie und \\ Hepatologie, Universitätsmedizin Mainz \\ 2 Centrum für chronische Immundefizienz - CCI, \\ Universitätsklinikum Freiburg, Medizinische Fakultät, \\ Albert-Ludwigs-Universität Freiburg \\ 3 Institut für Pathologie, Universitätsmedizin Mainz
}

Jan-Christopher Metzger ${ }^{1 *}$, Elena Kurz ${ }^{1 *}$, Caroline von Spee-Mayer ${ }^{2}$, Gesa Kolck ${ }^{1}$, Alexander Bogumil ${ }^{3}$, Peter R. Galle ${ }^{1}$,

Schlüsselwörter

Septische Granulomatose, CED, Morbus Crohn, Leberabszess

Key words

chronic granulomatous disease, Crohn's disease, liver abscess, IBD

eingereicht 11.05.2018

akzeptiert 20.08.2018

\section{Bibliografie}

DOI https://doi.org/10.1055/a-0732-5865

Online-Publikation: 22.11.2018

Z Gastroenterol 2018; 56: 1507-1512

(c) Georg Thieme Verlag KG, Stuttgart · New York

ISSN 0044-2771

Korrespondenzadresse

Prof. Dr. Tim Zimmermann

I. Medizinische Klinik und Poliklinik, Gastroenterologie und Hepatologie, Universitätsmedizin der Johannes Gutenberg Universität Mainz, Langenbeckstrasse 1, 55131 Mainz

Tel.: ++49/6131/173980

Fax: ++49/6131/17476075

tim.zimmermann@unimedizin-mainz.de

\section{ZUSAMMENFASSUNG}

Hintergrund Die septische Granulomatose ist eine seltene Granulozytenfunktionsstörung mit einer Prävalenz von etwa 150 Fällen in Deutschland. In der Literatur sind nur wenige Fälle mit einer intestinalen Manifestation, die als eine chronisch-entzündliche Darmerkrankung (CED) imponiert, beschrieben. Durch eine gestörte Superoxid-Bildung kommt es zu

* Geteilte Erstautorenschaft zwischen Jan-Christopher Metzger und Elena Kurz.
Infektionen mit seltenen Erregern, die häufig in Form pulmonaler Infekte, Lymphadenopathien und Leberabszessen auftreten. Wir präsentieren den Fall eines 28-jährigen Patienten, bei dem bereits im Alter von 2 Jahren die Diagnose einer CED gestellt wurde, der sich aktuell mit dem Bild einer therapierefraktären CED und rezidivierenden Leberabszessen vorstellte.

Methoden Patientendaten, Laborwerte, endoskopische, radiologische und histologische Befunde wurden erhoben. Granulozytenfunktionstests sowie eine genetische Analyse wurden durchgeführt. Es erfolgte eine selektive Literaturrecherche in der PubMed-Datenbank. Die aktuell verfügbare Literatur wurde zusammengefasst.

Fallbericht Unter langjähriger Immunsuppression, zuletzt mit dem TNF-Blocker Adalimumab und im Anschluss mit dem darmselektiven Integrin-Antagonist Vedolizumab kam es zu rezidivierenden Leberabszessen mit Nachweis eines gut sensiblen Staphylococcus aureus im Abszesspunktat. In der Koloskopie zeigten sich eine Rektumstenose sowie eine mäßige entzündliche Aktivität. Angesichts eines fehlenden Ansprechens der intestinalen Symptomatik auf sämtliche CED-Therapieversuche, einer floriden Akne und rezidivierenden, kaum kontrollierbaren, hepatischen Abszessen mit nicht primär intestinalen Erregern, stellten wir den Verdacht auf eine Granulozytenfunktionsstörung. In der Durchflusszytometrie sowie im oxidativen Burst-Test bestätigte sich die Diagnose einer septischen Granulomatose. Die genetische Untersuchung ergab eine homozygote Mutation des auf Chromosom 7 liegenden p47phox (NCF1)-Gens, welche mit 20-25\% die häufigste autosomal-rezessiv vererbte Form darstellt.

Ergebnisse Der Fall zeigt vor dem Hintergrund der aktuellen Literatur, dass besonders bei jungen Patienten mit therapieresistenten CED-ähnlichen Krankheitsbildern in Kombination mit infektiösen Komplikationen durch nicht darmtypische Erreger differenzialdiagnostisch an eine septische Granulomatose zu denken ist.

\section{ABSTRACT}

Background Chronic granulomatous disease is a rare disease with a prevalence of approximately 150 cases in Germany. An intestinal manifestation that mimics chronic inflammatory bowel disease (IBD) has only been described in a few cases. As a result of a deficient superoxide-synthesis, frequent and 
recurrent infections caused by rare pathogens have been described. We present the case of a 28-year old patient who has been diagnosed with IBD at the age of 2 years. He showed recurrent liver abscesses and the picture of a chronic IBD.

Methods Clinical and laboratory data was obtained and endoscopic, radiologic and histologic examinations, tests for granulocytic functions as well as a genetic analysis were performed. Literature of the PubMed database and recent literature were analyzed.

Case Under immunosuppressive therapy, with TNF -blocker Adalimumab followed by therapy with integrin-receptor antagonist Vedolizumab, the patient developed recurrent abscesses of the liver. Those were the result of infection with a sensitive Staphylococcus aureus strain. Colonoscopy showed stenosis of the rectum and some inflammatory activity. Intes- tinal symptoms were unresponsive to all therapies for IBD. Furthermore, there was a presence of active acne and recurrent liver abscesses due to bacteria not typical for intestinal infections. Consequently, we considered a granulocyte dysfunction as the underlying cause. Diagnosis of a chronic granulomatous disease was confirmed by flow cytometry and oxidative burst test. Genetic analysis showed a homozygote mutation of the p47phox (NCF1) gene located on chromosome 7 , which represents the most common autosomal recessive form with $20-25 \%$ of cases.

Results In light of recent literature, this case report shows that chronic granulomatous disease should be considered as a differential diagnosis to therapy refractory IBD. This is the case, especially in young patients, when recurrent bacterial lesions caused by intestine-atypical pathogens appear.

\section{Einleitung}

Die septische Granulomatose ist eine seltene Erkrankung mit einer Inzidenz von etwa 1:200 000 Neugeborenen, die mit einer Granulozytenfunktionsstörung einhergeht.

Neben der häufigsten Form, welche X-chromosomal rezessiv vererbt wirbt, gibt es 4 weitere autosomale Mutationen, welche in einem Defekt der NADPH-Oxidase der phagozytären Zellen resultieren [1]. Hierdurch kommt es zu einer gestörten Superoxid-Bildung, wodurch insbesondere die Abwehr gegen Katalasepositive Bakterien (Staphylococcus aureus, Gram-negative Enterokokken) und Pilze (Aspergillus fumigatus) beeinträchtigt ist [2]. Dies führt gehäuft zu Infektionen mit seltenen Erregern, von denen einige fast schon pathognomonisch für eine chronisch granulomatöse Erkrankung sind. Hierzu zählen u.a. Chromobacterium violaceum, Francisella philomiragia, Granulibacter bethesdensis und Burkholderia gladioli [3]. Die Infektionen können alle Körperregionen betreffen, treten jedoch am häufigsten in Form pulmonaler Infekte, Lymphadenopathien, Abszessen (v. a. Leber) und kutanen Läsionen auf [4]. Die Anfälligkeit der Patienten gegenüber Viren ist nicht verändert.

\section{Fallvorstellung}

Wir berichten über den Fall eines 28-jährigen Patienten mit seit dem zweiten Lebensjahr bestehender abdomineller Symptomatik, insbesondere rezidivierende Schmerzen, Stuhlunregelmäßigkeiten mit Diarrhöen, einer Akne und einer mikrozytären, hypochromen Anämie. Bei V. a. CED wurden über die Jahre mehrere Koloskopien mit Stufenbiopsien durchgeführt. Hierbei fanden sich im gesamten Kolon CED-typische Veränderungen: Kryptenarchitekturstörungen, diffuse, granuläre Rötungen, Kontaktblutungen, Ulzera, Pseudopolypen und Pflastersteinrelief. Makroskopisch und histologisch war die Abgrenzung der Befunde im Hinblick auf einen Morbus Crohn oder eine Colitis ulcerosa nicht eindeutig, sodass die Diagnose einer Colitis indeterminata gestellt wurde.

Bei einem Schub im Jahre 2008 wurde eine Therapie mit Mesalazin (Salofalk ${ }^{\circledR}$ ) durchgeführt. Daraufhin entwickelte der Patient einen hepatischen Abszess, welcher operativ ausgeräumt wurde. Als Erreger wurde ein gut sensibler Staphylococcus aureus festgestellt. In der Folge wurde Mesalazin abgesetzt. Bei erneuter histologischer Aktivität im Jahre 2015 wurde der Patient auf eine AntiTNF-Therapie mit Adalimumab eingestellt. Daraufhin entwickelte sich erneut ein Leberabszess, der drainiert wurde. Wieder war als ursächlicher Erreger ein Staph. aureus zu eruieren. Die Adalimumab-Gabe wurde beendet.

Im März 2017 wurde eine Kontrollkoloskopie durchgeführt und eine ausgeprägte Kolitisaktivität im gesamten Kolon sowie eine Rektumstenose von $2 \mathrm{~cm}$ ab ano festgestellt. Es erfolgten drei Gaben von Infliximab, worauf der bekannte hepatische Abszess rezidivierte und antibiotisch behandelt wurde. Infliximab wurde daraufhin eingestellt und stattdessen eine Therapie mit Vedolizumab, einem darmselektiven Integrin-Antagonist, begonnen. Der Patient reagierte mit einem neuen hepatischen Abszess, welcher drainiert und mit Meropenem behandelt wurde. Die Therapie mit Vedolizumab wurde beendet. Keine der bis dahin unternommenen Versuche, die rezidivierenden Colitisschübe zu behandeln, zeigte Wirkung.

Im September 2017 stellte sich der Patient mit starken Oberbauchschmerzen, Gewichtsverlust, Nachtschweiß und Fieber vor. Es wurden zwei neue Abszesse und ein Rezidiv eines bereits vorbekannten Abszesses festgestellt. Andere Infektionsherde konnten ausgeschlossen werden. Die Abszesspunktion war erfolgreich. Der Patient wurde, bei ausstehendem mikrobiologischem Befund, breit antibiotisch mit Ciprofloxacin und Meropenem abgedeckt, entfieberte jedoch nicht.

Als Ursache der rezidivierenden infektiösen Läsionen wurde bei chronisch-entzündlicher Darmerkrankung mit gestörter mukosaler Barrierefunktion eine Streuung der Erreger aus dem Darm über das Pfortadersystem in die Leber vermutet. Deshalb erfolgte die Verlegung des Patienten in unsere Klinik zur Evaluation der Sinnhaftigkeit einer Kolektomie.

Bei der Vorstellung zeigte sich ein 28-jähriger Patient in gutem Allgemein- und Ernährungszustand (180 cm Körpergröße, 82 kg Körpergewicht; BMI 25,3) mit abdominellen Schmerzen und Stuhlunregelmäßigkeiten (bei Aufnahme 1- bis 2-mal täglich 
Stuhlgang ohne Blutbeimengungen, rezidivierende Episoden mit Diarrhöen). In der klinischen Untersuchung zeigte sich eine Akne insbesondere im Bereich der Wangen bei ansonsten unauffälligen Befunden. Die Laboruntersuchung zeigte die bekannte hypochro-

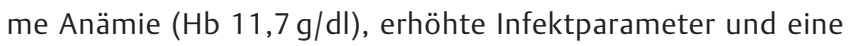
deutliche Erhöhung der IgE und IgA-Werte (IgE: $709 \mathrm{IU} / \mathrm{ml}$, Normwert: < $100 \mathrm{IU} / \mathrm{ml}$, IgA: 5,58 g/l, Normwert: 0,63 - 4,84 g/l) (weitere Laborparameter siehe Anhang). Der CDAI (Crohn's disease activity index) lag bei 111 entsprechend eines asymptomatischen Patienten. Wir führten erneut eine Sonografie und eine MRT des Abdomens durch. Diese zeigten multiple hepatische Abszesse verschiedener Größe (Segmente II, III, V, VI, VIII) ( mikrobiologischen Untersuchungen des Abszesspunktates ergaben erneut einen gut sensiblen Staph. aureus als Auslöser. Außerdem wurde eine Abszessstraße entlang des Stichkanals zur Drainage der Leberabszesse festgestellt, welche ebenfalls mit dem gleichen Bakterium besiedelt war. Unter intravenöser Therapie mit Flucloxacillin entfieberte der Patient und die Entzündungsparameter waren adäquat regredient. Nach operativer Abszessausräumung und täglicher Spülung und unter antibiotischer Therapie verheilte die Abszessstraße folgenlos. Die hepatischen Abszesse waren ebenfalls größenregredient.

Die durchgeführte Koloskopie zeigte eine diffuse Schleimhautrötung, multiple Narben und wenige Pseudopolypen im gesamten Kolon. Makroskopisch entsprach der Befund einer geringgradig aktiven Pankolitis ( $\mathbf{A} \mathbf{b} \mathbf{b} . \mathbf{2}$ ). In der histologischen und mikrobiologischen Untersuchung konnte eine infektiöse Genese ausgeschlossen werden. Es wurden Kryptenarchitekturstörungen, eine Stromafibrose und Eosinophilen-Infiltrate festgestellt $(\triangleright$ Abb. 3).

Aufgrund des für den Gastrointestinaltrakt eher untypischen Erregers der rezidivierenden, hepatischen Abszesse, der floriden Akne und des bisherigen Nicht-Ansprechens auf jegliche Behandlungsversuche der CED vermuteten wir als gemeinsame Ursache der gastrointestinalen Beschwerden und rezidivierenden Infektionen einen Immundefekt. Bei unauffälligem Differenzialblutbild mit normwertiger Neutrophilenzahl, Serum-Elektrophorese und Immunglobulinsynthese, konnte ein Defekt der Granulopoese und der humoralen Immunabwehr weitgehend ausgeschlossen werden. Weitere Ursachen, insbesondere eine HIV-Infektion, wurden ebenso ausgeschlossen. Es blieb die Möglichkeit einer Granulozytenfunktionsstörung. Bei Verdacht auf eine septische Granulomatose (chronic granulomatous disease, CGD) wurde eine Durchflusszytometrie durchgeführt. In dieser wird nach Stimulation mit Dihydrorhodamine 123 (DHR) und Phorbol-12Myristat-13-Acetat (PMA) das Ansprechen der Granulozyten gemessen. Eine fehlende adäquate Antwort bestätigte bei unserem Patienten die Verdachtsdiagnose einer septischen Granulomatose ( Abb.4).

Daraufhin wurde der Patient prophylaktisch mit Cotrimoxazol und Itraconazol zur Vorbeugung opportunistischer bakterieller und mykotischer Infektionen behandelt [5]. Eine genetische Diagnostik wurde veranlasst, um den Subtyp der Erkrankung festzustellen. Diese ergab eine homozygote Mutation des auf Chromosom 7 liegenden p47phox (NCF1) -Gens. Mit 20-25\% aller CGD-Fälle stellt diese Mutation die häufigste der autosomal-rezessiv vererbten Formen dar [3, 5]. Im CYBB-Gen, welches auf dem

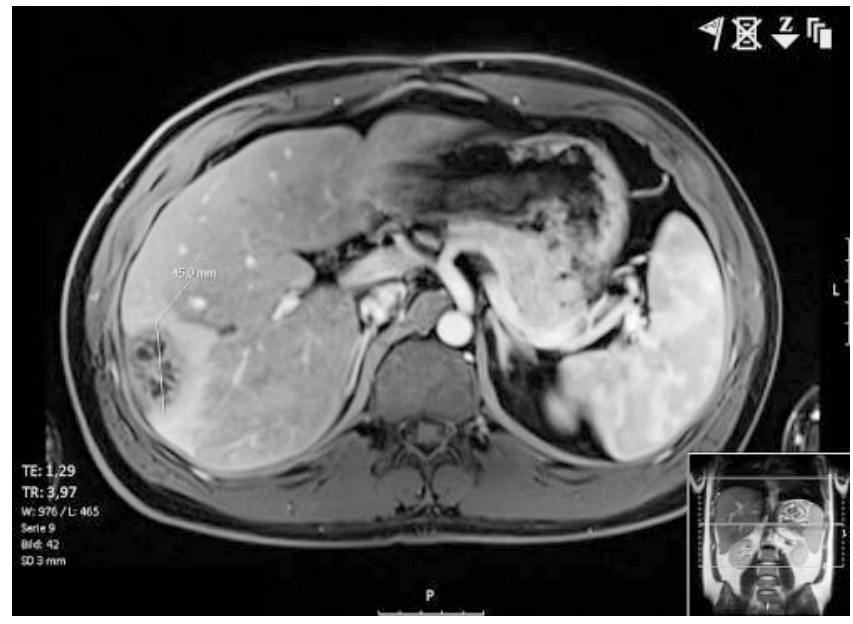

- Abb. 1 MRT-Abdomen mit Leberabszess, Durchmesser 45 mm, T1-Wichtung.

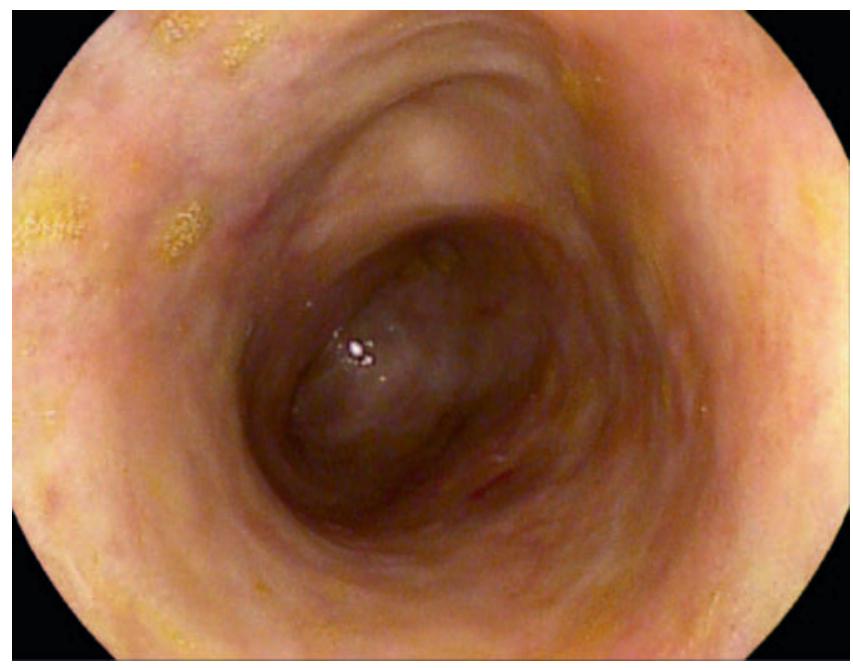

- Abb. 2 Koloskopie mit Bild einer geringgradig aktiven Pankolitis.

X-Chromosom liegt und mit ca. 60 \% für die meisten Krankheitsfälle verantwortlich ist, fanden sich keine Veränderungen. Im Anschluss erfolgte eine Anbindung an ein spezialisiertes Centrum für Chronische Immundefizienz und eine hämatologische Weiterbetreuung mit der Evaluierung einer allogenen Stammzelltransplantation.

\section{Diskussion}

Aufgrund der Seltenheit der CGD finden sich zu diesem Thema nur wenige Publikationen. Randomisiert-prospektive Studien fehlen fast gänzlich, lediglich Jaggi et al. untersuchten anhand eines CED-Kollektivs prospektiv den Nutzen eines Screenings auf CGD [6]. Barbato et al. berichten von einem 5 Jahre alten Jungen, der neben unspezifischen gastrointestinalen Symptomen insbesondere durch Hautläsionen aufgefallen war [7]. Er wurde als M. Crohn behandelt, bis in den Hautläsionen der Erregernachweis von Serratia marcescens gelang. Die Autoren folgerten, dass insbe- 

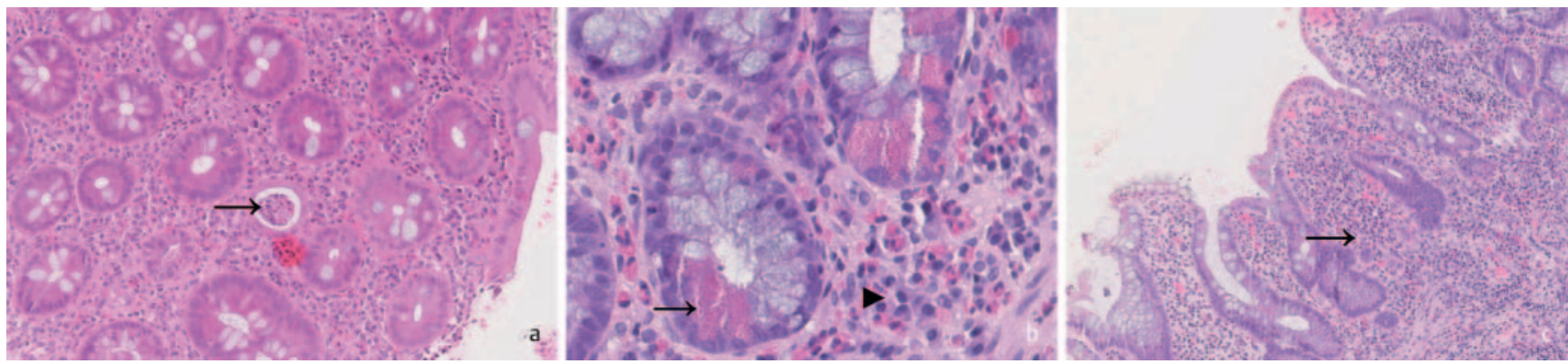

- Abb. 3 a Histologie; Architekturgestörtes Kolon mit aktiver Entzündung, destruierte Krypte mit Kryptenempyem (Pfeil). b Histologie; PanethMetaplasie (Pfeil) und Eosinophilen-Infiltrate (Spitze). c Histologie; Übersicht mit Störung der Kryptenarchitektur und epitheliale Regeneration (Pfeil).

sondere aufgrund der Seltenheit des Crohns bei Kindern in solchen Fällen auch die CGD als potenzielle Differenzialdiagnose in Betracht zu ziehen sei. Bei einem zweiten, ebenfalls 5-jährigen Jungen traten seit frühester Kindheit rezidivierende Infekte auf, dazu rektale Blutabgänge, Diarrhöen und Abszesse am Hals [8]. Erst der Nachweis von Burkholderia gladioli im Wundsekret des Abszesses führte zur Diagnose der CGD. Huang et al. berichten von einem 10-jährigen Mädchen, das an einer therapierefraktären Colitis vermeintlich im Rahmen eines M. Crohn litt [9]. Erst als eine Aspergillose des zentralen Nervensystems hinzukam, wurde die Diagnose einer CGD gestellt [10].

Dass - wie bei unserem Patienten - die Erkrankung erst mit 28 Jahren diagnostiziert wird, ist selten. So berichten Winkelstein et al., dass nur bei etwa $4 \%$ der Patienten die Diagnose im 3. Lebensjahrzehnt gestellt wird [2]. In einer Analyse des National Institute of Health (NIH) von 140 CGD-Patienten, fanden sich bei einem Drittel gastrointestinale Symptome, welche bereits bei einem medianen Alter von 2 Jahren zur Diagnose CGD führten [10]. Interessanterweise wird auch hier von 3 Patienten berichtet, welche zuerst fälschlicherweise mit der Diagnose Morbus Crohn behandelt wurden. Bei 2 dieser Patienten führte eine positive Familienanamnese zur Diagnose CGD.

Interessanterweise wurde über unseren Patienten bereits in früher Kindheit eine Kasuistik veröffentlicht [11]. Er wurde als seltener Fall einer bereits im 2. Lebensjahr auftretenden Colitis ulcerosa beschrieben und anhand seines Verlaufes wurde beispielhaft die Diagnostik und die Therapie chronisch-entzündlicher Darmerkrankungen im Kindesalter beschrieben. Aus heutiger medizinischer Sicht ist der Fall retrospektiv kritisch zu hinterfragen. So weisen Uhlig et al. explizit darauf hin, dass bei Erstdiagnose einer CED im frühen Kindesalter immer auch Immundefizienzen als Differenzialdiagnosen ausgeschlossen werden müssen [12]. Dies erscheint bei unserem Patienten umso naheliegender, nachdem er über Jahrzehnte nur inadäquat auf die immunsuppressive Therapie angesprochen hatte.

Neben der CGD gibt es viele weitere monogenetische Varianten, die CED-ähnliche Symptome bereits im frühen Kindesalter hervorrufen können. Bei diesen Patienten sollte frühzeitig eine genetische Analyse durchgeführt werden [12]. Aufgrund der Fülle der möglichen Mutationen und der deutlich verbesserten Verfügbarkeit wird in diesen Fällen heutzutage auch die Möglichkeit der Sequenzierung des gesamten Genoms diskutiert.
Noch später als bei unserem Patienten wurde die Diagnose im Fall einer 53-jährigen Patientin gestellt, die eine Vorgeschichte mit M. Crohn und multiplen Leberabszessen aufwies [13]. Erst als sie eine Pneumonie mit Burkholderia cepacia entwickelte, konnte die korrekte Diagnose gestellt werden. Es ist davon auszugehen, dass milde CGD-Formen deutlich unterdiagnostiziert sind.

Ein häufiges Symptom, das sowohl bei CED als auch bei der CGD in der Kindheit auftreten kann, ist die Gedeihstörung. 32\% der Patienten des oben genannten NIH-Kollektivs lagen zumindest teilweise unterhalb der 5. Perzentile des Längenwachstums, während dies bei unserem Patienten nicht der Fall ist. Dies entspricht auch den Angaben von Buescher et al., die von einem oft verzögerten Wachstum bei CGD-Patienten berichten, welche dies in der späteren Adoleszenz aufholen und eine durchschnittliche Höhe erreichen [14]. Marks et al. berichten von 28 CGD Patienten, die derzeit in ihrem Zentrum in London betreut werden. Davon zeigte sich bei 11 (44\%) das Bild einer CED mit medianem Symptombeginn mit 14 Jahren [15]. Auch hier gab es 3 Patienten, die zuerst als M. Crohn diagnostiziert wurden. Die Ursache hierfür liegt sicher in den teils identischen, oft unspezifischen Symptomen wie Bauchschmerzen, Diarrhöen und Gedeihstörungen. Aber auch bei spezifischeren Symptomen ähneln sich die Krankheitsbilder: skip lesions, Granulome, Fisteln und Abszesse können bei beiden Entitäten auftreten. Jedoch gibt es auch Unterschiede: So befällt die CGD meist - wie auch bei unserem Patienten - das Rektum, selten das terminale lleum, geht öfter als der Morbus Crohn mit Abszessen einher und führt häufiger zu Fieber, Husten und einer Lymphadenopathie [16]. In der Analyse von 368 CGDPatienten fanden sich bei 98 Leberabszesse (27\%) [2]. Für eine sichere Unterscheidung der beiden Krankheitsbilder sind diese Kriterien jedoch sicher nicht ausreichend, weshalb Jaggi et al. den Nutzen eines Screenings untersuchten [6]. 120 CED Patienten wurden auf CGD getestet. In ihrem Kollektiv fand sich kein Betroffener, weshalb die Autoren schlossen, dass ein Screening auf CGD bei einer solch seltenen Krankheit nicht sinnvoll ist. Jedoch kann man kritisch anmerken, dass dieses Screening nicht bei Kindern oder jugendlichen CED-Patienten durchgeführt wurde.

In der Therapie der CGD liegt wie bei anderen Immunschwächen der Fokus auf der Infektionsprophylaxe, welche das Überleben der Patienten in den letzten Jahrzehnten signifikant verlängert hat [17]. Diese besteht aus der lebenslangen Gabe von Cotrimoxazol ( $120 \mathrm{mg}$ bei Patienten < 6 Monaten bis $960 \mathrm{mg}$ bei 




Abb.4 Durchflusszytometrie mit fehlender Antwort der Granulozyten auf Stimulation mit DHR und PMA im Vergleich zu gesunder Kontrollperson; Sicherung der Diagnose CGD.

$>12$ Jahren) und Itraconazol (5 mg/kg KG/d) [18]. Eine weitere therapeutische Option stellt die Prophylaxe mit IFN-gamma dar, deren Effektivität jedoch umstritten ist [19, 20].

Aufgrund der erhöhten Mortalität der CGD-Patienten bei Infektionen gilt die Maxime „hit fast and hard“ [2, 21]. Insbesondere bei nach 24-48 Stunden noch therapierefraktären Infektionen gilt es die antibiotische Therapie zu eskalieren und vorab unbedingt eine adäquate mikrobiologische Erregerdiagnostik mit Resistenztestung durchzuführen. Auch muss stets die Möglichkeit einer Pilzinfektion (insbesondere eine invasive Aspergillose) in Betracht gezogen werden, da deren Prävalenz bei CGD größer ist als bei jeder anderen angeborenen Immunschwäche [3]. Eine 
widersprüchliche Rolle in der Therapie bei CGD-Patienten spielen Kortikosteroide. Erscheint deren immunsuppressive Wirkung auf den ersten Blick kontraindiziert, so konnte durch ihren Einsatz sowohl die überschießende Entzündungsreaktion gedämpft als auch bei therapierefraktären Leberabszessen eine chirurgische Intervention vermieden werden [22]. Der Einsatz von Kortikosteroiden sollte jedoch nur in Kombination mit einer adäquaten antiinfektiven Therapie erfolgen.

Die derzeit einzige kausale therapeutische Option besteht in der allogenen hämatopoetischen Stammzelltransplantation [23]. Jedoch ist diese ebenfalls mit Risiken wie lebensbedrohlichen Komplikationen verbunden. Eine Alternative könnte die Gentherapie darstellen, welche jedoch bisher nur in klinischen Studien zur Anwendung kommt [24, 25].

Zusammenfassend muss die CGD als seltene Differenzialdiagnose zu einer CED, v. a. einem Morbus Crohn, in Betracht gezogen werden, wenn die Erstdiagnose im frühen Kindesalter lag. Verläufe mit jahrzehntelanger inadäquater Therapie sind möglich. Insbesondere bei multiplen Infektionen mit seltenen, für den Gastrointestinaltrakt eher untypischen Erregern, sollte an eine CGD gedacht werden. Je früher die richtige Diagnose gestellt wird, desto günstiger ist die Prognose für die Patienten, da mit einer adäquaten Infektionsprophylaxe und einem schnellen Eingreifen beim Auftreten von Infektionen die Mortalität in den letzten Dekaden signifikant reduziert werden konnte. Aufgrund der Seltenheit und Komplexität der Erkrankung ist die Anbindung an ein spezialisiertes Zentrum zu empfehlen.

\section{Interessenkonflikt}

Die Autoren geben an, dass kein Interessenkonflikt besteht.

\section{Literatur}

[1] Kang EM, Marciano BE, DeRavin S et al. Chronic granulomatous disease: overview and hematopoietic stem cell transplantation. | Allergy Clin Immunol 2011; 127: 1319-1326; quiz 27-28

[2] Winkelstein JA, Marino MC, Johnston RB Jr et al. Chronic granulomatous disease. Report on a national registry of 368 patients. Medicine (Baltimore) 2000; 79: 155-169

[3] Arnold DE, Heimall JR. A Review of Chronic Granulomatous Disease. Adv Ther 2017; 34: $2543-2557$

[4] Lee M, Lee MS, Lee JS et al. Spectrum of imaging findings of chronic granulomatous disease: a single center experience. Diagn Interv Radiol 2017; 23: 472-477

[5] The CGD Society, Dr Andrew Gennery, Professor Adrian Thrasher, North DN. Chronic granulomatous disorder. A guide for medical professionals. 2012

[6] Jaggi P, Scherzer R, Knieper R et al. Utility of screening for chronic granulomatous disease in patients with inflammatory bowel disease. J Clin Immunol 2012; 32: 78 -81
[7] Barbato M, Ragusa G, Civitelli F et al. Chronic granulomatous disease mimicking early-onset Crohn's disease with cutaneous manifestations. BMC Pediatr 2014; 14: 156

[8] Hoare S, Walsh JE, Eastham E et al. Abnormal technetium labelled white cell scan in the colitis of chronic granulomatous disease. Arch Dis Child 1997; 77: $50-51$

[9] Huang JS, Noack D, Rae J et al. Chronic granulomatous disease caused by a deficiency in p47(phox) mimicking Crohn's disease. Clin Gastroenterol Hepatol 2004; 2: 690-695

[10] Marciano BE, Rosenzweig SD, Kleiner DE et al. Gastrointestinal involvement in chronic granulomatous disease. Pediatrics 2004; 114: 462 - 468

[11] Hoppen T, Keller KM, Chalybäus C et al. Colitis ulcerosa - Diagnose, Therapie und Komplikationen im frühen Kindesalter. Hautnah Pädiatrie 1995; 7: 15-22

[12] Uhlig HH, Schwerd T, Koletzko S et al. The diagnostic approach to monogenic very early onset inflammatory bowel disease. Gastroenterology 2014; 147: 990 - 1007 e3

[13] Ramanuja S, Wolf KM, Sadat MA et al. Newly diagnosed chronic granulomatous disease in a 53-year-old woman with Crohn disease. Ann Allergy Asthma Immunol 2005; 95: 204-209

[14] Buescher ES, Gallin Jl. Stature and weight in chronic granulomatous disease. J Pediatr 1984; 104: 911-913

[15] Marks DJ, Miyagi K, Rahman FZ et al. Inflammatory bowel disease in CGD reproduces the clinicopathological features of Crohn's disease. Am J Gastroenterol 2009; 104: 117-124

[16] Damen GM, van Krieken JH, Hoppenreijs E et al. Overlap, common features, and essential differences in pediatric granulomatous inflammatory bowel disease. J Pediatr Gastroenterol Nutr 2010; 51: 690-697

[17] Margolis DM, Melnick DA, Alling DW et al. Trimethoprim-sulfamethoxazole prophylaxis in the management of chronic granulomatous disease. J Infect Dis 1990; 162: 723-726

[18] Gallin JI, Alling DW, Malech HL et al. Itraconazole to prevent fungal infections in chronic granulomatous disease. N Engl J Med 2003; 348: $2416-2422$

[19] Douglas B, Kuhns PDW, Gregory Alvord PD et al. A controlled trial of interferon gamma to prevent infection in chronic granulomatous disease. The International Chronic Granulomatous Disease Cooperative Study Group. N Engl ] Med 1991; 324: 509-516

[20] Martire B, Rondelli R, Soresina A et al. Clinical features, long-term followup and outcome of a large cohort of patients with Chronic Granulomatous Disease: an Italian multicenter study. Clin Immunol 2008; 126: $155-164$

[21] Marciano BE, Spalding C, Fitzgerald A et al. Common severe infections in chronic granulomatous disease. Clin Infect Dis 2015; 60: 1176-1183

[22] Leiding JW, Freeman AF, Marciano BE et al. Corticosteroid therapy for liver abscess in chronic granulomatous disease. Clin Infect Dis 2012; 54: $694-700$

[23] Kang EM, Malech HL. Gene therapy for chronic granulomatous disease. Methods Enzymol 2012; 507: 125-154

[24] Seger RA. Modern management of chronic granulomatous disease. $\mathrm{Br}$ J Haematol 2008; 140: 255-266

[25] Grez M, Reichenbach J, Schwable J et al. Gene therapy of chronic granulomatous disease: the engraftment dilemma. Mol Ther 2011; 19: 28-35 